\title{
PROGRAMA CIENTÍFICO PARA O MONITORAMENTO EM TEMPO REAL OU PÓS-PROCESSADO DAS IRREGULARIDADES IONOSFÉRICAS E CINTILAÇÃO DOS SINAIS GNSS
}

\author{
Scientific program for monitoring in real time or post-processed of ionospheric \\ irregularities and scintillation of GNSS signals
}

\author{
Vinícius Amadeu Stuani Pereira ${ }^{1}$ \\ Paulo de Oliveira Camargo ${ }^{1}$ \\ 1 Programa de Pós-Graduação em Ciências Cartográficas - FCT/UNESP. Departamento de Cartografia \\ - FCT/UNESP.Rua Roberto Simonsen, 305, CEP 19060-900, Presidente Prudente, São Paulo, \\ Brasil.Email:vi_stuani@hotmail.com, paulo@fct.unesp.br
}

\section{Resumo:}

O Brasil é um dos países que mais sofrem os efeitos provocados pela ionosfera, principalmente os oriundos da Anomalia de Ionização Equatorial, irregularidades ionosféricas e cintilação dos sinais GNSS (Global Navigation Satellite System). Várias estratégias podem ser utilizadas para minimizar os efeitos, tais como: modelos ionosféricos, arquivos IONEX ou a combinação linear ion-free. Em se tratando do monitoramento da ionosfera a situação é diferente. A quantidade de instrumentos dedicados ao estudo da camada ionosférica é reduzida no âmbito brasileiro. Neste contexto foi desenvolvido o programa científico denominado Ion_Index, com objetivo de estimar indicadores dos níveis de irregularidades da ionosfera e de cintilação dos sinais GNSS em tempo real ou pós-processado, utilizando a infraestrutura já existente de dados GNSS de redes ativas públicas, como a RBMC, a GNSS-SP e a CALIBRA, transformando assim estações GNSS em estações monitoras da camada ionosférica. Dessa forma é proporcionado um aumento na resolução espacial das informações sobre o comportamento da ionosfera na região brasileira, permitindo um melhor entendimento e contribuindo para o desenvolvimento ou aprimoramento de modelos de mitigação. Experimentos utilizando dados de ionossondas digitais e de receptores PolaRxS-PRO da Septentrio (fontes externas) comprovam a eficiência do programa.

Palavras-chave: Irregularidades ionosféricas. Cintilação ionosférica. Índices $\mathrm{f}_{\mathrm{P}}, \mathrm{F}_{\mathrm{P}}$, IROT, ROTI, $\sigma_{\Phi}, \mathrm{S}_{\Phi}$ e $\sigma_{\text {CHAIN. }}$ ROT.

\begin{abstract}
:
Brazil is one of the countries that suffer more the effects of the ionosphere, especially those from the Equatorial Ionization Anomaly, ionospheric irregularities and scintillation of GNSS (Global Navigation Satellite System) signals. Several strategies can be used to minimize the effects, such as: ionospheric models, IONEX files or ion-free linear combination. In the case of monitoring of the ionosphere the situation is different. The amount of instruments dedicated to the study of ionospheric layer is reduced in the Brazilian region. In this context it developed a scientific program, namely Ion_Index, in order to estimate indicators of levels of ionospheric irregularities
\end{abstract}


and scintillation of GNSS signals in real time or post-processed using the existing infrastructure of GNSS data public active networks, as the RBMC, GNSS-SP and CALIBRA, thus transforming GNSS stations in monitoring stations of the ionospheric layer. This way it is provided an increase in the spatial resolution of the information on the ionosphere behavior in the Brazilian region, allowing a better understanding and contributing to the development or enhancement of mitigation models. Experiments using digital ionosondes and PolaRxS-PRO Septentrio receivers data (external sources) prove the efficiency of the program.

Keywords: Ionospheric irregularities. Ionospheric scintillation. Indices $f_{P}, F_{P}, I_{R O T}, R O T I, \sigma_{\Phi}, S_{\Phi}$ and $\sigma_{\text {CHAIN. }}$ ROT.

\section{Introdução}

Um dos fatores que afeta o resultado do posicionamento GNSS (Global Navigation Satellite System) é a ionosfera, camada atmosférica localizada entre 50 e $1.000 \mathrm{~km}$ de altura, considerada a maior fonte de erro sistemático após a desativação da técnica SA (Selective Availability) em 2000. O efeito sistemático devido à ionosfera é diretamente proporcional ao Conteúdo Total de Elétrons (TEC - Total Electron Content) e inversamente proporcional ao quadrado da frequência do sinal. Uma característica importante é que o TEC varia no tempo e no espaço em razão do fluxo de ionização solar, atividade geomagnética, ciclo de manchas solares, estações do ano, hora local, direção do raio vetor do satélite e localização geográfica, sendo de difícil tratamento (Camargo et al., 2000; Leick, 1995). Devido ao efeito sistemático da ionosfera sobre os sinais GNSS ser de natureza dispersiva, pode-se a partir de receptores de dupla frequência eliminar o efeito de primeira ordem por meio de uma combinação linear entre as portadoras L1 e L2 (ionfree), bem como, calcular o TEC presente na ionosfera e o erro nas observáveis (pseudodistância e fase da onda portadora) (Seeber, 2003; Lin, 1997). Para minimizar o efeito da ionosfera no posicionamento por ponto e relativo em regiões com altos gradientes na variação do TEC, como o caso do Brasil, os usuários de receptores de uma frequência podem utilizar modelos ionosféricos globais ou regionais (Camargo et al., 2000).

Em consequência da alta radiação solar na região equatorial e da configuração do campo magnético da Terra, a densidade de elétrons nesta região sofre sensíveis efeitos, sendo um deles denominado de efeito fonte (Webster, 1993). O efeito fonte gera a Anomalia de Ionização Equatorial (AIE), que consiste em duas faixas de alta densidade do plasma ionosférico, localizadas nas regiões tropicais que circulam paralelamente ao Equador magnético. Nas faixas da anomalia que se localizam sobre o território brasileiro as densidades de elétrons atingem valores maiores do que sobre outras regiões da Terra. A maior intensidade ocorre nos dois lados do Equador magnético, entre as latitudes geomagnéticas de $\pm 10^{\circ} \mathrm{e} \pm 20^{\circ}$ (Rodrigues, 2003).

A variação na densidade de elétrons causa um efeito denominado de cintilação ionosférica, que por sua vez provoca variações de amplitude, de fase, no ângulo e na polarização do sinal, quando o mesmo atravessa uma região de irregularidades na densidade eletrônica (Klobuchar, 1996). As cintilações são fortes nas zonas aurorais e nas regiões tropicais durante o período noturno, próximos aos equinócios de primavera e outono, e a ocorrência da cintilação aumenta durante os períodos de alta atividade solar e outras atividades extremas tal como a ocorrência de tempestades geomagnéticas (Conker et al., 2003; Davies, 1990). No Brasil, as ocorrências de cintilações são mínimas de maio a agosto e máximas de setembro a abril. Os horários de cintilação mais intensos são limitados ao período entre o pôr do Sol até, aproximadamente, meia noite local, e têm o potencial de afetar todos os serviços GNSS (Fu et al., 1999).

Bol. Ciênc. Geod., sec. Artigos, Curitiba, v. 22, no2, p.282 - 302, abr - jun, 2016. 
Com os experimentos já realizados (Pereira e Camargo, 2013; Oladipo e Schuler, 2013; Chu et al., 2008; Shan et al., 2002) verifica-se que é possível realizar estudos do comportamento da camada ionosférica a partir de dados de redes ativas. Com esses dados pode-se calcular o TEC, o ROT (Rate of Change of TEC), índices de irregularidades da ionosfera, tais como: $\mathrm{f}_{\mathrm{P}} \mathrm{e} \mathrm{F}_{\mathrm{P}}$ (Mendillo et al., 2000), IROT (Pi et al., 1997) e ROTI (Wanninger, 1993), bem como, alguns índices específicos para o estudo da cintilação ionosférica: $\sigma_{\Phi}$ (Van Dierendonck et al., 1993), S (Forte, 2007) e $\sigma_{\text {CHAIN }}$ (Mushini et al., 2012); além disso gerar mapas de cintilação e das irregularidades, que permitem observar o comportamento espacial e temporal e entender o ciclo de atividades da ionosfera, possibilitando a mitigação dos efeitos da mesma, principalmente no posicionamento GNSS.

O Brasil dispõe de dados GNSS para a realização do monitoramento das irregularidades da ionosfera, uma vez que o IBGE (Instituto Brasileiro de Geografia e Estatística) estabeleceu a Rede Brasileira de Monitoramento Contínuo dos Sistemas GNSS (RBMC), que iniciou com nove estações espalhadas pelo Brasil coletando dados continuamente (Fortes, 1997). Atualmente a RBMC conta com mais de 115 estações. Os receptores GNSS da RBMC coletam dados a cada 15 segundos, que por sua vez são disponibilizados a cada 24 horas, exceto as estações da RBMC-IP (RBMC em Tempo Real - Internet Protocol), num total de 92, que os transmitem em tempo real, a cada segundo, via Ntrip (Networked Transport of RTCM via Internet Protocol). Também transmitem dados via Ntrip as 21 estações da rede GNSS-SP (Rede GNSS Ativa do Estado de São Paulo). Há também a rede CALIBRA (Countering GNSS high Accuracy applications Limitations due to Ionospheric disturbances in BRAzil), composta por 13 estações com receptores PolaRxS-PRO da Septentrio.

Considerando a importância do monitoramento das irregularidades e da cintilação da ionosfera, foi desenvolvido um programa científico (Ion_Index, disponível em <http://www.fct.unesp.br/\#!/ pesquisa/grupos-de-estudo-e-pesquisa/gege/softwares/>) com objetivo de estimar indicadores dos níveis de irregularidades da ionosfera e de cintilação dos sinais GNSS para o território brasileiro utilizando dados GNSS das redes ativas públicas CALIBRA, GNSS-SP e RBMC, tanto no modo pós-processado quanto, principalmente, em tempo real, haja vista que várias aplicações necessitam de informações dessa natureza (agricultura de precisão, aproximação e pouso de aeronaves, etc.), uma vez que a quantidade de instrumentos dedicados ao monitoramento da ionosfera no Brasil é reduzida, tais como ionossondas, imageadores, radares de espalhamento coerente e receptores do tipo PolaRxS-PRO da Septentrio.

\section{2. Índices de irregularidades da ionosfera e de cintilação dos sinais GNSS}

As irregularidades ionosféricas são perturbações na densidade do plasma ionosférico, que se originam por meio de processos de instabilidade de plasma do tipo Rayleigh-Taylor (R-T) que ocorrem na ionosfera tropical. A instabilidade R-T dá origem às irregularidades na camada $\mathrm{F}$ ionosférica com um amplo espectro de escalas de tamanho, que vão desde alguns centímetros até centenas de quilômetros. Já as cintilações ionosféricas são flutuações da amplitude e/ou fase de uma onda eletromagnética, resultado da sua propagação através de uma região na qual existem irregularidades na densidade de elétrons e, consequentemente, do índice de refração (Rodrigues, 2003). A cintilação causa um enfraquecimento no sinal captado pelos receptores GNSS, fazendo com que ocorra em muitos casos a perda do sinal (Webster, 1993). 
As estimativas dos índices de irregularidades ( $f_{P}, F_{P}, I_{R O T}$ e ROTI) se baseiam na taxa de variação do TEC (ROT). O ROT é calculado por meio da razão da diferença entre os TECs obtidos consecutivamente entre duas épocas $(\triangle T E C)$, pelo intervalo de tempo decorrido entre as respectivas determinações $(\Delta \mathrm{t})$ :

$$
R O T=\frac{T E C_{t_{z}}-T E C_{t_{s}}}{t_{2}-t_{1}}=\frac{\Delta T E C}{\Delta t} .
$$

O TEC pode ser obtido utilizando tanto as pseudodistâncias advindas dos códigos nas portadoras L1 e L2 quanto a partir da combinação linear entre as medidas de fase das portadoras L1 e L2 (Matsuoka e Camargo, 2004):

$$
T E C_{r}^{s}=\frac{f_{1}^{2} f_{2}^{2}}{40,3\left(f_{l}^{2}-f_{2}^{2}\right)}\left[\left(\lambda_{2} \Phi_{2 r}^{s}-\lambda_{1} \Phi_{l r}^{s}\right)-\left(\lambda_{1} N_{l r}^{s}-\lambda_{2} N_{2 r}^{s}\right)-e_{\Phi_{l 2}}\right],
$$

onde $\mathrm{f}_{1}$ e $\mathrm{f}_{2}$ são as frequências das ondas portadoras $\mathrm{L} 1$ e $\mathrm{L} 2, \lambda_{1}$ e $\lambda_{2}$ os comprimentos das ondas portadoras L1 e L2, $\Phi_{1 \mathrm{r}}^{\mathrm{s}}$ e $\Phi_{2 \mathrm{r}}^{\mathrm{s}}$ as medidas de fase em ciclos das ondas portadoras L1 e L2, $\mathrm{N}_{1 \mathrm{r}}^{\mathrm{s}}$ e $\mathrm{N}_{2 \mathrm{r}}{ }_{\mathrm{r}}^{\mathrm{s}}$ as ambiguidades da fase das portadoras L1 e L2, e $\mathrm{e}_{\Phi_{12}}$ os erros sistemáticos não eliminados na combinação linear e erros aleatórios.

Substituindo a Equação 2 na Equação 1 pode-se obter uma estimativa do ROT tendo como parâmetro apenas as medidas de fase das portadoras L1 e L2 nas épocas $t_{1}$ e $t_{2}$ :

$$
R O T=\frac{9,52 \cdot 10^{16}\left[\lambda_{2}\left(\Phi_{2 r_{t_{z}}}^{s}-\Phi_{2 r_{t_{t}}}^{s}\right)-\lambda_{1}\left(\Phi_{l_{r_{t_{z}}}}^{s}-\Phi_{l_{r_{t_{t}}}}^{s}\right)\right]}{t_{2}-t_{1}} .
$$

A Equação 3 é utilizada para os satélites GPS (Global Positioning System), os quais se baseiam na técnica de identificação dos satélites denominada CDMA (Code Division Multiple Access) (Hofmann-Wellenhof et al., 2008). Já para o GLONASS (Global'naya Navigatsionnaya Sputnikovaya Sistema), que utiliza divisões múltiplas de frequências para diferenciar cada satélite (FDMA - Frequency Division Multiple Access) (Hofmann-Wellenhof et al., 2008), devese adotar a Equação 4 , sendo $\mathrm{f}_{1}, \mathrm{f}_{2}, \lambda_{1}$ e $\lambda_{2}$ as frequências e comprimentos de onda referentes às portadoras L1 e L2 de cada satélite observado:

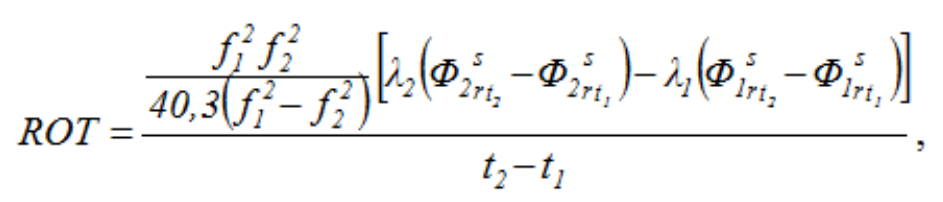

Wanninger (1995) destaca que após a determinação do ROT, utilizando a fase da onda portadora, alguns erros podem não ser minimizados ou eliminados totalmente, como o caso do multicaminho, que não ultrapassa $0,3 \cdot 10^{16} \mathrm{~m}^{-2} \mathrm{~min}^{-1}$.

$\mathrm{O}$ índice de irregularidades $\mathrm{f}_{\mathrm{P}}$ (phase fluctuation) é o valor da mediana dos ROTs para um período de 15 minutos, sendo os ROTs estimados a cada minuto. O índice é calculado para cada satélite de uma determinada estação/receptor (Mendillo et al., 2000):

$$
f_{P}(n, h r, i)=\text { Mediana }|R O T|,
$$

onde n é o número do satélite, hr a hora cheia (de 0 a 24h TU (Tempo Universal)) e i o número da seção, com duração de 15 minutos, contida em uma hora cheia, ou seja, i = 1, 2, 3 ou 4. De acordo com Mendillo et al. (2000), o índice representa a resolução espacial das irregularidades ionosféricas. 
$\mathrm{O}$ índice $\mathrm{F}_{\mathrm{P}}$ é estimado para cada estação e representa o valor médio dos índices $\mathrm{f}_{\mathrm{P}}$ obtidos para todos os satélites observados em uma estação no período de uma hora, que por sua vez é obtido por meio da Equação 6 (Mendillo et al., 2000):

$$
F_{P}(h r)=\frac{\sum_{n}^{n s a t}\left[\sum_{i}^{k} f_{P}(n, h r, i) / k \mid\right.}{n s a t(h r)} 1000
$$

onde nsat é o número total de satélites observados durante o período de uma hora e $\mathrm{k}$ o número de valores de $f_{P}$ disponíveis em cada hora $(\mathrm{k}=0,1,2,3$ ou 4).

A utilização do índice destina-se a retratar o nível geral de irregularidades ionosféricas presentes na vizinhança de uma determinada estação. Um valor $F_{P} \leq 50$ representa baixos níveis de irregularidades; $50<\mathrm{F}_{\mathrm{P}} \leq 200$ significa a presença de irregularidades moderadas, e quando $\mathrm{F}_{\mathrm{P}}>$ 200 representa a ocorrência de fortes níveis de irregularidades ionosféricas (Mendillo et al., 2000).

Wanninger (1993) apresenta o índice $\mathrm{I}_{\text {ROT }}$ para caracterizar flutuações de fase como diagnóstico de irregularidades ionosféricas, sendo calculado para um período de 15 minutos com base no RMS (Root Mean Square) dos ROTs:

$$
I_{R O T}=10 \cdot R M S(R O T) \text {. }
$$

Diferentemente de Mendillo et al. (2000), Wanninger (1993) não apresenta um padrão de classificação do índice $\mathrm{I}_{\mathrm{ROT}}$; ele apenas conclui que valores próximos ou superiores a 3 caracterizam fortes irregularidades ionosféricas. Assim, Pereira e Camargo (2014) realizaram experimentos visando determinar um padrão para o $\mathrm{I}_{\mathrm{ROT}}$, obtendo os seguintes limiares: $\mathrm{I}_{\mathrm{ROT}} \leq$ 0,5 - baixos níveis de irregularidades; $0,5<$ IROT $_{\text {R }} \leq 2,0$ - irregularidades moderadas; e $\mathrm{I}_{\mathrm{ROT}}>2,0$ - níveis de irregularidades ionosféricas muito fortes.

Devido ao fato de que as flutuações da fase em pequena escala não estavam sendo identificadas nos índices existentes, Pi et al. (1997) sugerem que um índice para a taxa de variação do TEC poderia ser determinado com base no desvio-padrão do ROT em um intervalo de cinco minutos. Este índice é denominado de ROTI, e pode ser obtido da seguinte forma:

$$
R O T I=\sqrt{\left\langle R O T^{2}\right\rangle-\langle R O T\rangle^{2}}
$$

onde $<>$ representa a média.

Análoga à situação do índice $\mathrm{I}_{\mathrm{ROT}}$, um padrão para o índice ROTI teve que ser determinado: $\mathrm{ROTI} \leq 0,05$ representa baixos níveis de irregularidades; $0,05<\mathrm{ROTI} \leq 0,2$ significa a presença de irregularidades moderadas, e quando ROTI > 0,2 representa a ocorrência de fortes níveis de irregularidades ionosféricas (Pereira e Camargo, 2014).

A propagação dos sinais GNSS através de regiões de irregularidades ionosféricas podem ocasionar reflexões nos mesmos, acarretando em rápidas flutuações na intensidade dos sinais, denominadas cintilações de amplitude (Conker et al., 2003). As cintilações de amplitude são quantificadas pelo índice S4, que consiste no desvio-padrão normalizado das observações da intensidade do sinal livres de tendências, as quais são amostradas em altas taxas (geralmente 50 Hz) em um intervalo de 60 segundos (Conker et al., 2003; Van Dierendonck et al., 1993). As tendências referem-se aos efeitos que o sinal sofre durante o percurso do satélite ao receptor que não são classificados como cintilação, como por exemplo, o erro da órbita e fase wind-up do satélite, erros do relógio do receptor e do satélite, multicaminho, ruídos do receptor, dentre outros (Niu, 2012; Monico, 2008). 
Já as cintilações de fase acontecem a partir de rápidas variações na fase do sinal, quando o mesmo percorre as irregularidades na densidade de plasma da ionosfera. Fortes cintilações de fase podem provocar perdas de ciclos, bem como, a perda de sintonia entre o receptor e satélite (Walter et al., 2010). Essas cintilações são quantificadas pelo índice $\sigma_{\Phi}$ (Equação 9), que consiste no desvio-padrão das observações da fase do sinal livres de tendências $\left(\Phi_{\mathrm{e}}\right)$, as quais são coletadas a altas frequências $(50 \mathrm{~Hz})$ em um intervalo de 60 segundos, sendo às vezes conhecido como $\Phi_{60}$ (Van Dierendonck et al., 1993).

$$
\sigma_{\Phi}=\sqrt{\left\langle\Phi_{e}^{2}\right\rangle-\left\langle\Phi_{e}\right\rangle^{2}}
$$

$\mathrm{O}$ índice $\sigma_{\Phi}$ é dado em radianos, podendo ser expresso também em graus e metros. Hegarty et al. (2001) apresentam o seguinte padrão classificatório para a L1: cintilação muito fraca - 0,05 rad $<\sigma_{\Phi} \leq 0,2 \mathrm{rad}$; cintilação fraca $-0,2 \mathrm{rad}<\sigma_{\Phi} \leq 0,3 \mathrm{rad}$; cintilação moderada $-0,3 \mathrm{rad}<\sigma_{\Phi} \leq 0,6$ $\mathrm{rad}$; e cintilação forte $-\sigma_{\Phi}>0,6 \mathrm{rad}$.

O índice de cintilação de fase $S_{\Phi}$ foi proposto por Forte (2007) como uma alternativa para o índice $\sigma_{\Phi}$, devido a incertezas que existem sob o tradicional índice de cintilação de fase, conforme apresentado por Beach (2006) apud Niu (2012). O índice $S_{\Phi}$, dado em rad/s, é calculado pela expressão:

$$
S_{\Phi}=\sqrt{\left.\left(\frac{\partial \Phi_{e}}{\partial t}\right)^{2}\right)}
$$

Ao contrário do índice $\sigma_{\Phi}$, o $\mathrm{S}_{\Phi}$ leva em consideração a taxa de variação das flutuações de fase da onda portadora, mostrando, de acordo com Forte (2007), mais consistência quando se utiliza diferentes métodos de detrending (processo de remoção das tendências) das observações.

O índice de cintilação de fase CHAIN, introduzido por Mushini et al. (2012) e representado por $\sigma_{\text {CHAIN }}$ é resultado de uma modificação no índice $S_{\Phi}$ :

$$
\sigma_{C H A I N}=\sqrt{\left(\left(\frac{\partial \Phi_{e}}{\partial t}\right)^{2}\left|\Phi_{e}\right|\right)}
$$

O índice $\sigma_{\text {CHAIN }}$ utiliza, além da taxa de variação das flutuações da fase, também a amplitude das flutuações. É considerado um dos melhores índices de cintilação de fase para medidas realizadas em regiões de altas latitudes, se comparado com o $\sigma_{\Phi}$, uma vez que gera grande correlação com o índice de cintilação de amplitude S4 (Mushini et al., 2012; Niu, 2012).

\section{Programa para o monitoramento das irregularidades ionosféricas e cintilação dos sinais GNSS}

O fluxograma do Ion_Index é esquematizado na Figura 1, sendo dividido em duas modalidades: pós-processamento (Ion_Index_PP) e tempo real (Ion_Index_TR), ambas implementadas em linguagem $\mathrm{C}$. 


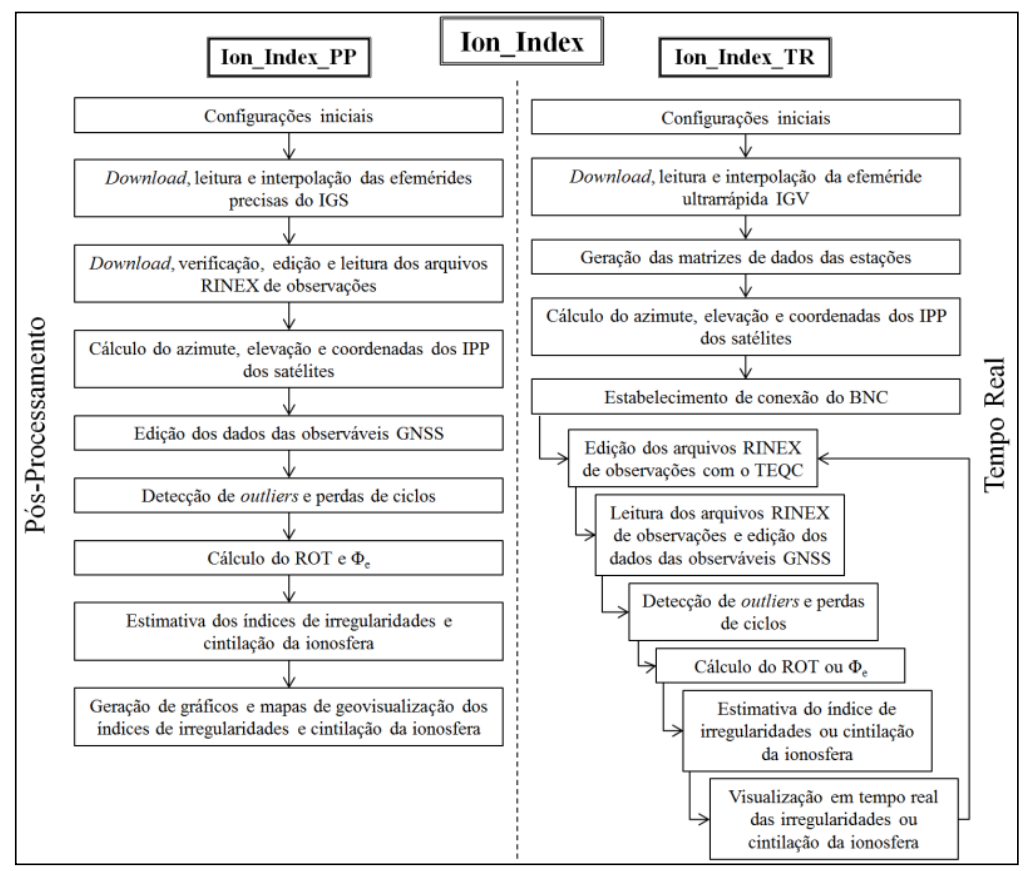

Figura 1: Fluxograma do Ion_Index.

Ressalta-se que o IPP (Ionospheric Pierce Point) consiste no ponto onde o sinal transmitido pelo satélite intercepta a camada ionosférica em uma dada altura média. A efeméride ultrarrápida IGV contém as coordenadas cartesianas dos satélites GLONASS e GPS.

\subsection{Modalidade - Pós-Processamento}

Para o monitoramento da ionosfera, no modo pós-processado, o usuário deve fornecer informações por meio da interface do programa, tais como: ano de processamento, dia e mês ou intervalo de processamento, rede(s) ativa(s), estação(s), índice(s) de irregularidades e/ou de cintilação da ionosfera, opção de gerar ou não mapas de geovisualização e a máscara de elevação.

Sete opções de combinações de redes ativas podem ser escolhidas: RBMC (Figura 2a); GNSSSP (Figura 2c); CALIBRA (Figura 2d); RBMC e GNSS-SP; RBMC e CALIBRA; GNSS-SP e CALIBRA; ou todas as redes. Em relação aos índices, pode-se escolher em estimar todos os índices de irregularidades e de cintilação da ionosfera, ou somente os índices de irregularidades, ou apenas os de cintilação, ou ainda informa o(s) índice(s). Caso o número de estações seja maior ou igual a dois, é requisitado se deseja gerar mapas de geovisualização. 


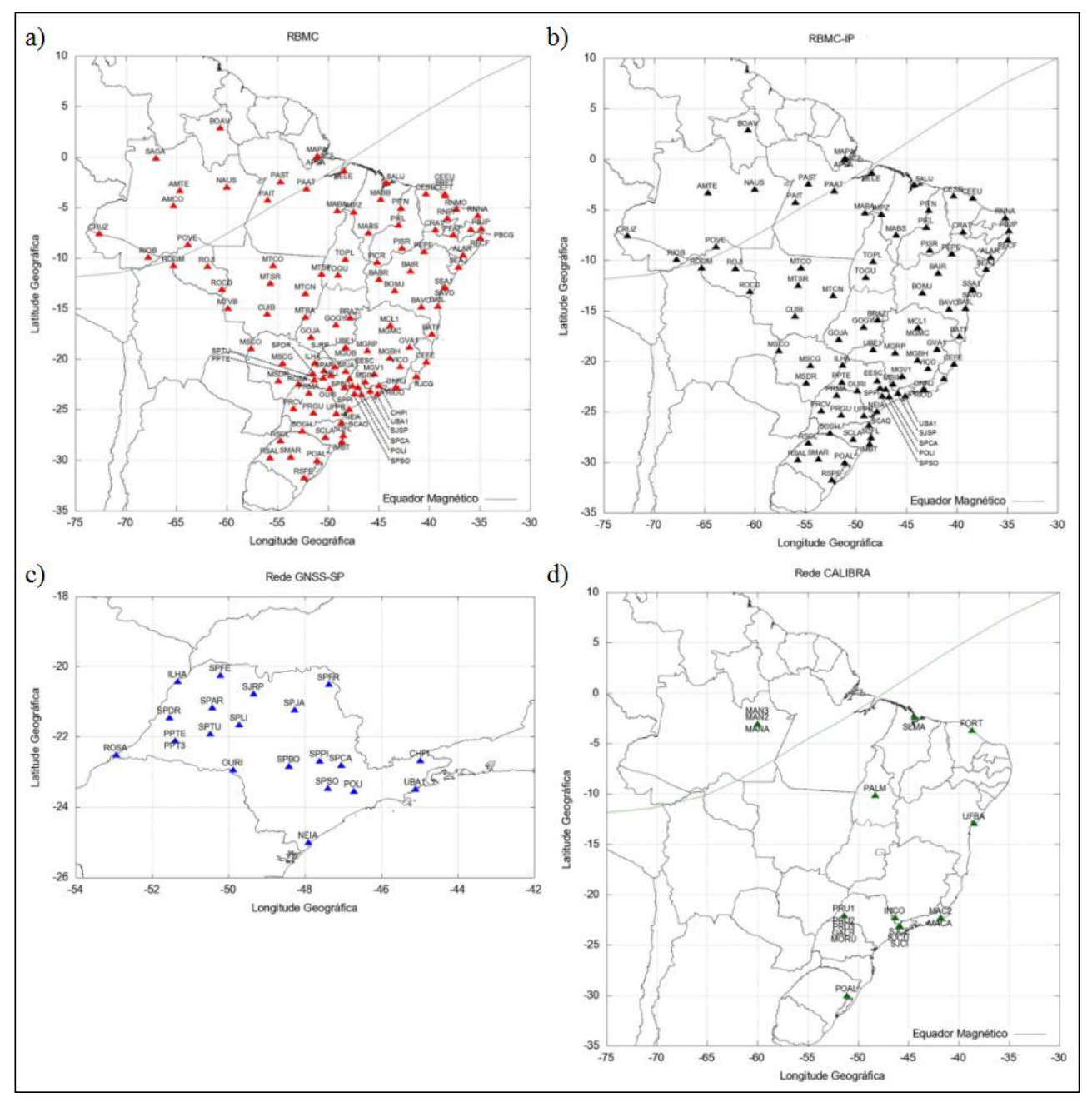

Figura 2: a) Estações da RBMC, b) RBMC-IP, c) GNSS-SP e d) CALIBRA.

Para cada uma das estações das redes ativas selecionadas são realizados os downloads dos arquivos de observações por meio dos FTPs dos servidores das redes. A rede CALIBRA disponibiliza os arquivos RINEX (Receiver Independent Exchange Format) de observações por meio do FTP <ftp://is-cigala-calibra.fct.unesp.br>, a rede GNSS-SP em <ftp://200.145.185.225/> e a RBMC pelo <ftp://geoftp.ibge.gov.br/RBMC/dados/>. Com o intuito de otimizar a leitura dos arquivos RINEX de observações é realizado uma edição dos arquivos utilizando o TEQC. As funções aplicadas são: exclusão dos sinais que não sejam GPS e GLONASS, exclusão das observáveis GNSS que não sejam a fase da onda portadora e pseudodistância nas portadoras L1 e L2, exclusão de comentários e ordenação crescente dos satélites para cada época. A respeito das efemérides, o Ion_Index_PP utiliza as precisas do IGS (International GNSS Service), disponíveis no FTP <ftp.igs.org/pub/product>.

Uma vez realizada a leitura da fase da onda portadora e da pseudodistância é efetuada uma edição preliminar nos dados antes da aplicação do algoritmo de Blewitt (1990) para detecção de outliers e perdas de ciclos: aplica-se a máscara de elevação e verifica-se para satélites GPS e GLONASS de cada época se as quatro medidas (fase e pseudodistância em L1 e L2) estão disponíveis. Caso não haja as quatro medidas concomitantemente o programa as excluem do conjunto de observações, pois para a detecção de outliers e perdas de ciclos é necessário que todas elas estejam disponíveis. No caso de perda de ciclo a fase da onda portadora apresenta um salto por um número inteiro de ciclos, afetando assim o cálculo do ROT e $\Phi_{\mathrm{e}}$. Logo, na 
ocorrência de perdas de ciclos, esses devem ser detectados para que o cálculo do ROT e $\Phi_{\mathrm{e}}$ não seja efetuado nos instantes das perdas.

O cálculo do ROT para os satélites GPS é realizado utilizando a Equação 3, considerando o intervalo de tempo de um minuto. Já para os satélites GLONASS aplica-se a Equação 4, também $\operatorname{com} \Delta \mathrm{t}=1 \mathrm{~min}$.

Com exceção do índice $\mathrm{S} 4$, os demais índices de cintilação da ionosfera são obtidos a partir do uso das observações da fase do sinal livres de tendências $\left(\Phi_{\mathrm{e}}\right)$. Vários métodos de detrending podem ser encontrados na literatura: filtragem Butterworth, ajuste polinomial, filtragem pela Transformada Contínua de Wavelet, filtragem pela Transformada Discreta de Wavelet, etc. Entretanto, a maioria desses métodos foram desenvolvidos para serem executados considerando medidas de fase amostradas em altas frequências, da ordem de $50 \mathrm{~Hz}$, os quais aplicam uma frequência de corte de $0,1 \mathrm{~Hz}$ para separar os componentes de cintilação dos não-cintilação. Em se tratando das medidas de fase provenientes dos arquivos RINEX das estações das redes GNSSSP, CALIBRA e RBMC, a frequência de amostragem é de apenas 0,067 Hz (uma observação a cada 15 segundos), o que inviabiliza a aplicação de qualquer um dos métodos de detrending. Sendo assim, a obtenção dos valores de $\Phi_{\mathrm{e}}$ se restringiu em apenas remover os efeitos de multicaminho nas medidas de fase a partir da aplicação de uma máscara de elevação, bem como, detectar e não considerar as observações em que ocorrem perdas de ciclos.

Com base nos valores de ROT são estimados os índices de irregularidades da ionosfera $\mathrm{f}_{\mathrm{P}}, \mathrm{F}_{\mathrm{P}}$, $\mathrm{I}_{\mathrm{ROT}}$ e ROTI, por meio das Equações 5, 6, 7 e 8, respectivamente. Em relação aos índices de cintilação, o Ion_Index_PP estima somente os indicadores das cintilações de fase $\sigma_{\Phi}$ (Equação 9), $S_{\Phi}$ (Equação 10) e $\sigma_{\text {CHAIN }}$ (Equação 11), haja vista que os receptores não fornecem a intensidade do sinal em seus arquivos RINEX de observações. No entanto, análises de cintilações de amplitude podem ser realizadas utilizando a ferramenta web ISMR Query Tool (http://is-cigala-calibra.fct.unesp.br/is/ismrtool/index.php).

Para a geração de gráficos e mapas de geovisualização dos índices é utilizado o GNUPLOT. Para os índices de irregularidades são gerados mapas de acordo com as resoluções temporais dos mesmos. Já para os índices de cintilação, que são estimados a cada minuto, são produzidos mapas com intervalos de cinco minutos.

\subsection{Modalidade - Tempo Real}

Na modalidade tempo real algumas etapas são congêneres da pós-processada: cálculo do azimute, elevação e coordenadas dos IPP dos satélites; edição dos arquivos RINEX de observações com o TEQC; leitura dos arquivos RINEX de observações e edição dos dados das observáveis GNSS; detecção de outliers e perdas de ciclos; cálculo do ROT ou $\Phi_{\mathrm{e}}$; e estimativa do índice de irregularidades ou de cintilação da ionosfera.

Diferentemente do modo pós-processado, onde todas as estações das três redes ativas podem ser selecionadas para o processamento, contabilizando um total aproximado de 130 estações, no modo tempo real há disponíveis as 92 estações da RBMC-IP (Figura 2b) e as 21 estações da GNSS-SP (Figura 2c); a rede CALIBRA não disponibiliza os dados via Ntrip. Em relação a qual índice de irregularidades ou de cintilação da ionosfera deseja-se estimar, o Ion_Index_TR realiza o processamento de apenas um índice por vez. Outras informações devem ser informadas pelo usuário, como o período de processamento, o fuso horário da localidade e o nome da pasta pessoal do computador. 
O processamento em tempo real se inicia com a conexão do BNC (BKG Ntrip Client). Nessa etapa é gerado um script "BNC.bnc" contendo todos os parâmetros de conexão com o Caster da(s) rede(s) ativa(s) selecionada(s). Uma vez que os arquivos RINEX de observações são criados e atualizados pelo BNC, optou-se por editá-los utilizando o TEQC, onde arquivos temporários contendo apenas as observáveis da época corrente são gerados. Esse método visa eliminar problemas de leitura que possam vir a surgir, problemas quanto à correspondência temporal entre todos os arquivos de observações das estações, e a corriqueira falha na transmissão das observáveis GNSS de alguma estação para um determinado período de tempo.

A etapa de detecção de outliers e perdas de ciclos é igual a do modo pós-processado, com algumas adaptações no algoritmo de Blewitt (1990) para o funcionamento em tempo real.

Com base nos valores de ROT é estimado o índice de irregularidades da ionosfera de interesse ou, caso tenha sido selecionado algum dos índices de cintilação, utiliza-se das medidas da fase do sinal livres de tendências. No entanto, para que os índices sejam estimados a cada segundo aplica-se o conceito de janela deslizante, sendo a janela de acordo com o período de dados específicos de cada índice e o deslize de 1 segundo. Desse modo, dependendo do índice escolhido, o programa permanece em espera até que o primeiro valor seja obtido, por exemplo: para o índice FP o Ion_Index_TR permanece 1 hora calculando e armazenando os valores de ROT até que o primeiro valor $F_{P}$ seja estimado, ao contrário do índice $\sigma_{\Phi}$, cujo tempo de espera é de apenas 1 minuto. Após o período de espera o GNUPLOT é executado automaticamente e a visualização em tempo real do mapa dos valores do índice de irregularidades ou de cintilação é gerada.

\section{Produtos gerados pelo programa e validação}

Três tipos de produtos podem ser obtidos por meio do Ion_Index_PP: arquivo texto contendo os valores numéricos do índice, gráfico de barras verticais para os índices de irregularidades da ionosfera ou gráfico de pontos para os índices de cintilação, e mapas de geovisualização para o monitoramento e análise geográfica das irregularidades e cintilação ionosférica.

Para exemplificar, a Figura 3 apresenta mapas de geovisualização do índice horário F $F_{P}$ referente ao período compreendido entre $21 \mathrm{~h}$ TU do dia 20/12/2014 (DOY (Day of Year) 354) e 5h TU do dia 21/12/2014 (DOY 355), período esse caracterizado por uma alta variabilidade da densidade eletrônica devido à proximidade do solstício de verão no hemisfério Sul. Para a geração dos mapas foram utilizadas todas as estações disponíveis das redes CALIBRA, GNSS-SP e RBMC, adotando uma máscara de elevação de $35^{\circ}$. O ponto preto em destaque na Figura 3 refere-se a localização da estação BRAZ (Brasília/DF) da RBMC. 


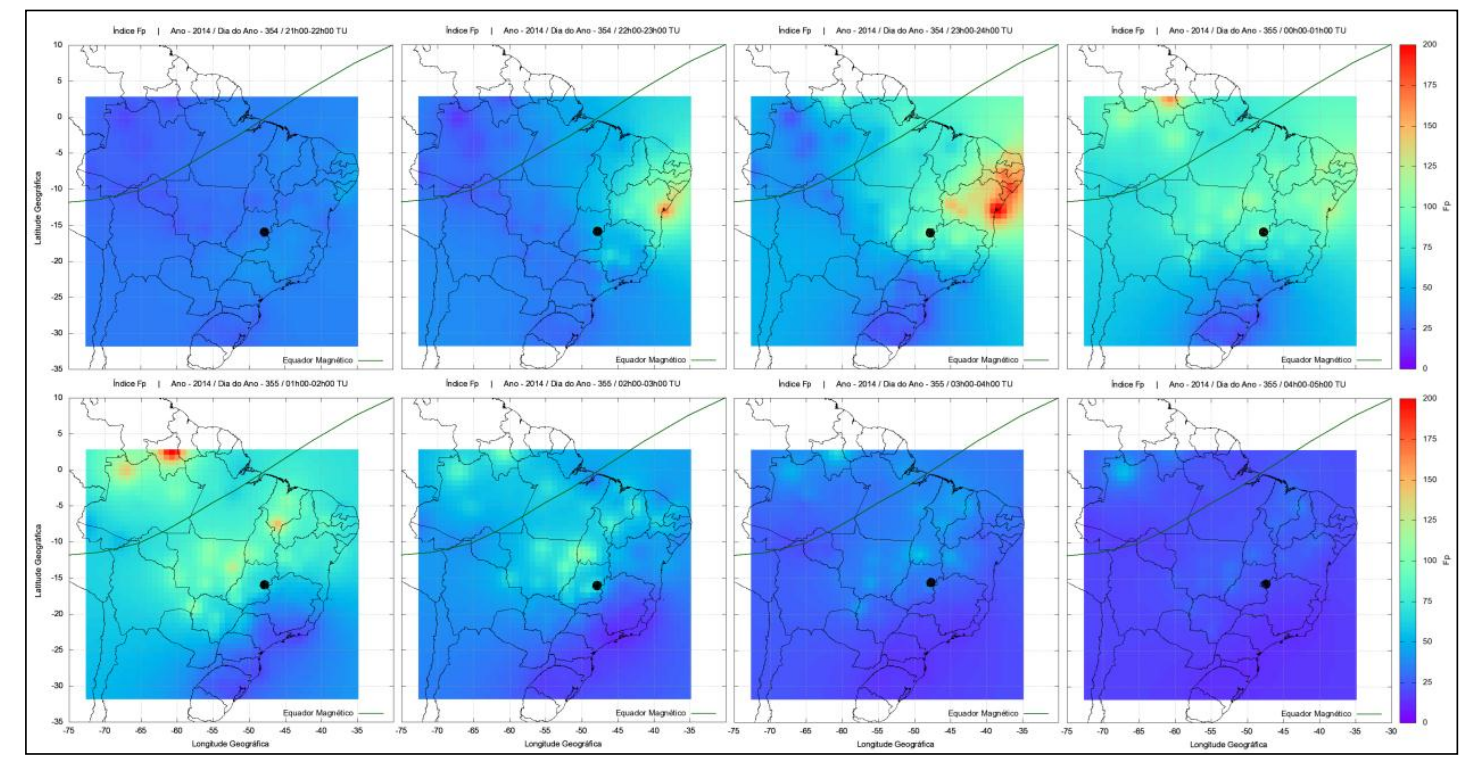

Figura 3: Mapas do índice $F_{P}$, período 21-05h TU dos dias 20 e 21/12/2014.

Uma das desvantagens em relação ao uso do índice FP no monitoramento das irregularidades da ionosfera se dá pela resolução temporal do mesmo, uma hora, impossibilitando um melhor acompanhamento das irregularidades. Utilizando os índices $f_{\mathrm{P}}$, IROT e ROTI pode-se obter uma melhor compreensão da evolução das irregularidades devido ao menor intervalo de tempo para obtenções dos índices. Nesse sentido são apresentados nas Figuras 4 e 5 mapas de geovisualização dos índices fP e $I_{R O T}$ para o período 22-24h TU do dia 20/12/2014. Em relação às cintilações ionosféricas de fase, a Figura 6 apresenta mapas do índice $\sigma_{\Phi}$ (portadora L1) para 0 período $23 \mathrm{~h} 20 \mathrm{~min}$ a $24 \mathrm{~h}$ TU do dia 20/12/2014.

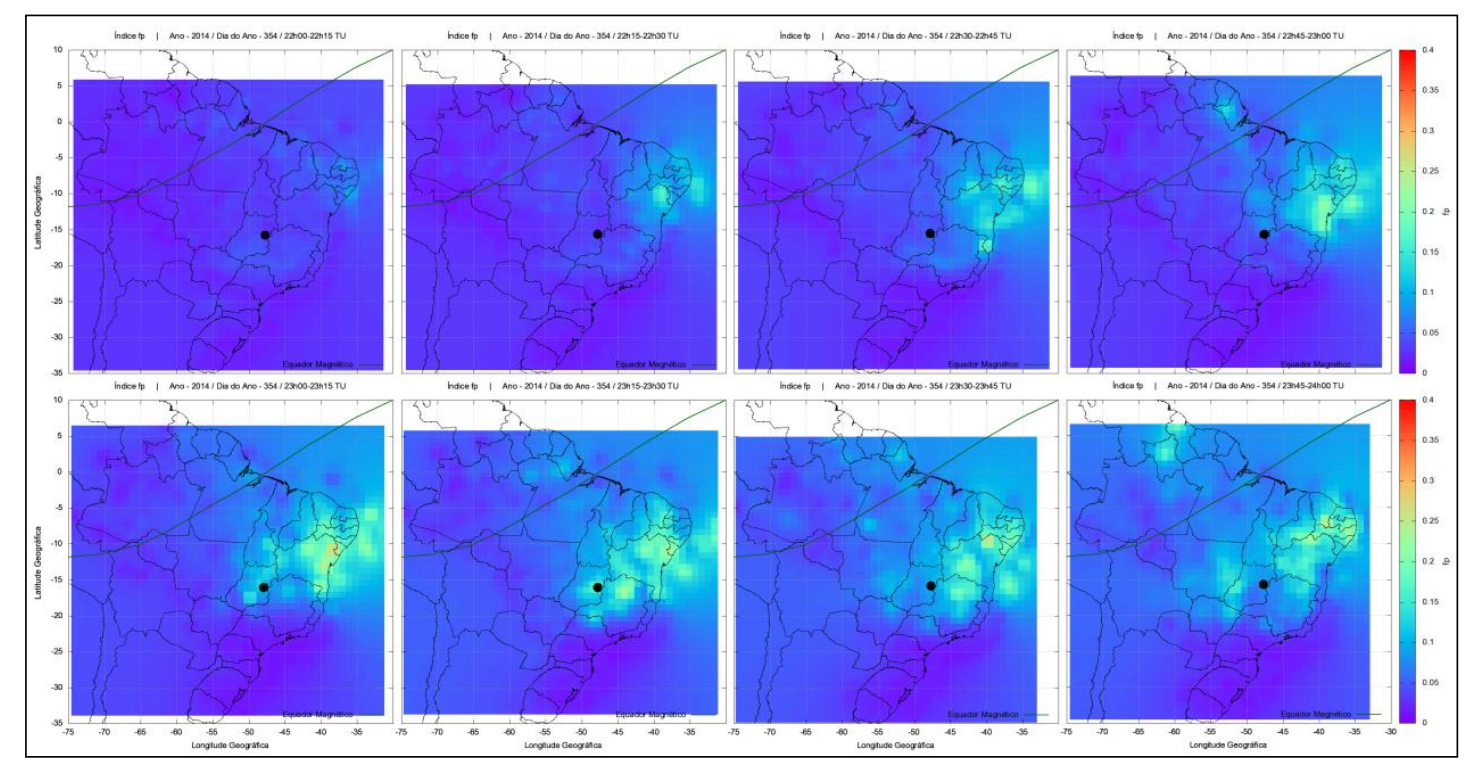

Figura 4: Mapas do índice fP, período 22-24h TU do dia 20/12/2014. 


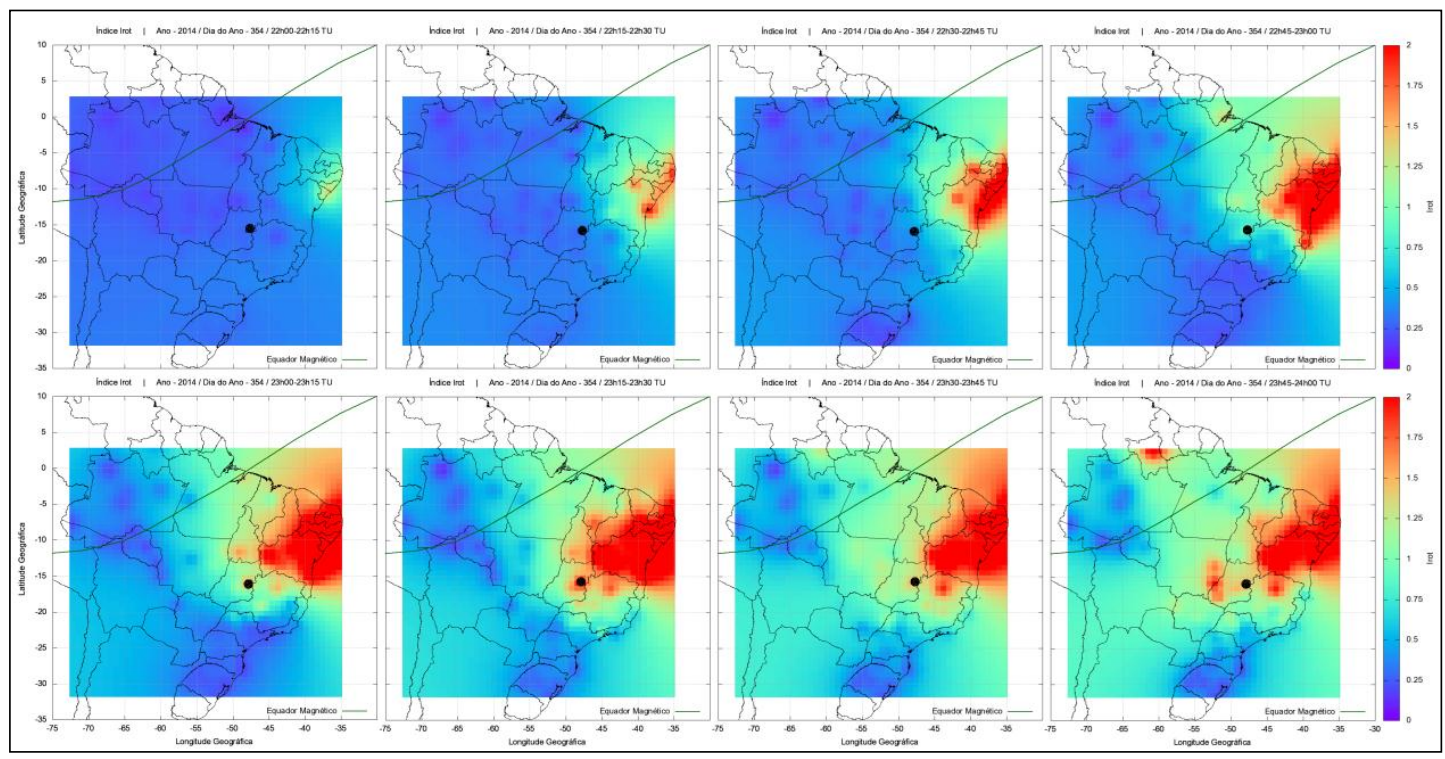

Figura 5: Mapas do índice $\mathrm{I}_{\mathrm{ROT}}$, período 22-24h TU do dia 20/12/2014.

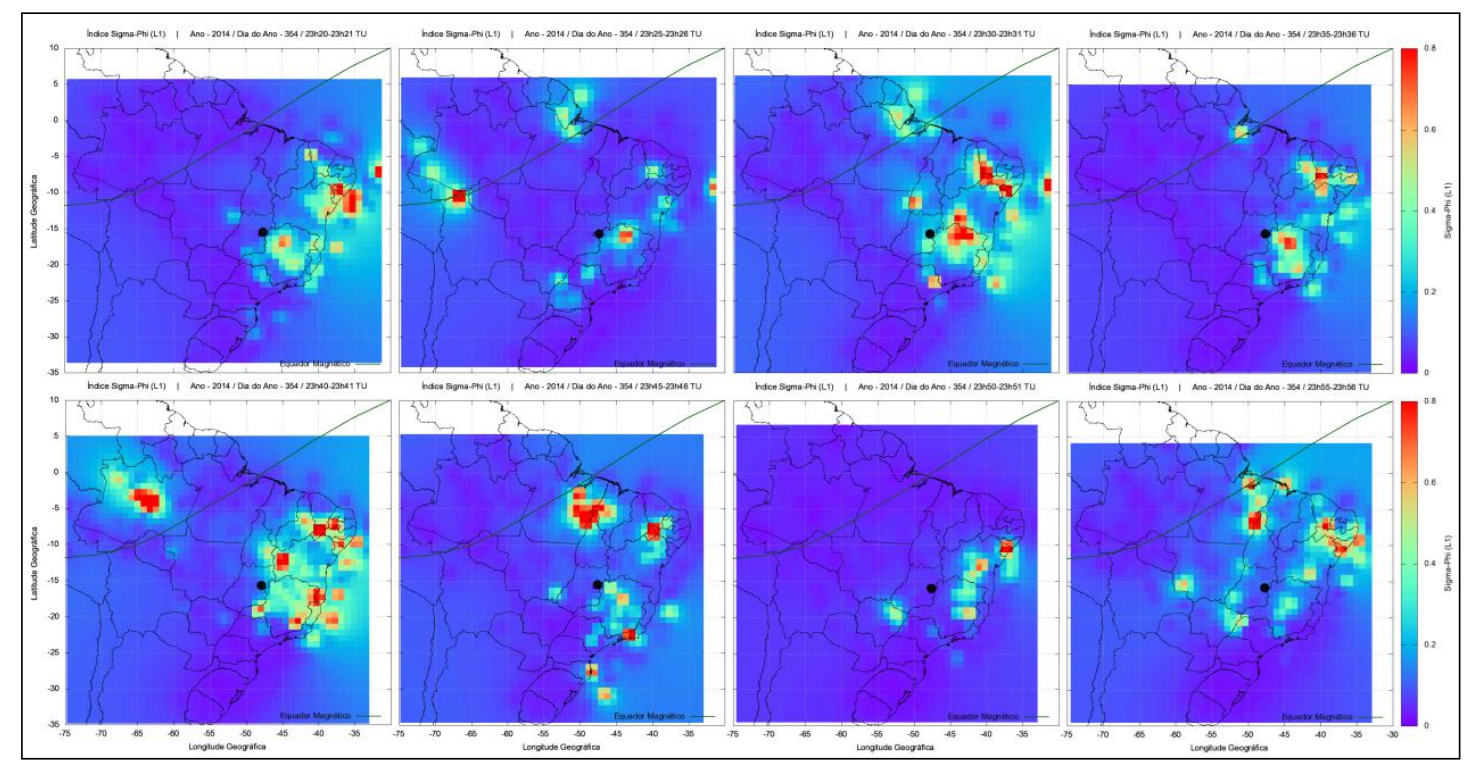

Figura 6: Mapas do índice $\sigma_{\Phi}$, período 23h20min a 24h TU do dia 20/12/2014.

Observando as Figuras 4 e 5 verifica-se a ocorrência de fortes irregularidades ionosféricas nas regiões Norte, Nordeste, Centro-Oeste e Sudeste, tendo início após o ocaso solar. Assim os sinais GNSS, ao atravessarem essas regiões de irregularidades na densidade do plasma ionosférico, sofrem cintilação ionosférica, conforme mostra a Figura 6. Selecionando a estação BRAZ para o dia 20/12/2014, como exemplo, pode-se obter os gráficos individuais de cada um dos índices ( $f_{\mathrm{P}}$, $F_{p}, I_{R O T}, R O T I, \sigma_{\Phi}, S_{\Phi}$ e $\sigma_{\text {CHAIN }}$ ) (Figura 7 ). Nos gráficos a linha verde indica o limite entre baixos e moderados níveis de irregularidades da ionosfera ou de cintilação dos sinais GNSS, ao passo que a linha vermelha indica o limite entre os níveis moderados e fortes.

Os valores numéricos dos índices podem ser obtidos acessando os arquivos texto que acompanham os gráficos. Para exemplificação, a Figura 8 apresenta alguns valores dos índices $f_{P}, F_{P}, \sigma_{\Phi}$ e $S_{\Phi}$ da estação BRAZ para o dia 20/12/2014. 


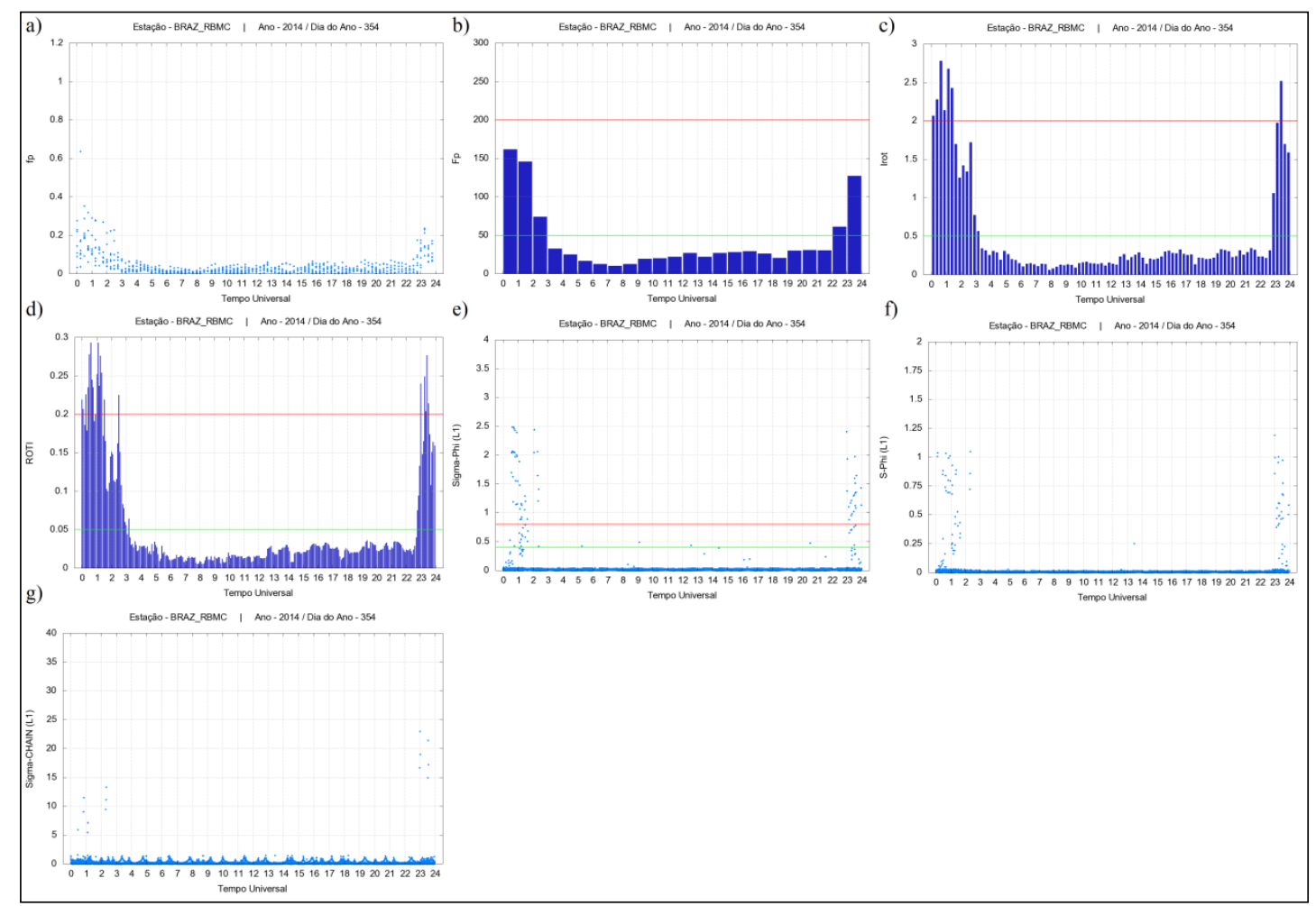

Figura 7: Gráficos dos índices a) $f_{P}$, b) $F_{P}$, c) $I_{R O T}$, d) ROTI, e) $\sigma_{\Phi}$, f) $S_{\Phi}$ e g) $\sigma_{C H A I N}$, BRAZ/RBMC, 20/12/2014.

\begin{tabular}{|c|c|c|c|c|c|c|c|c|c|c|c|c|c|}
\hline \multicolumn{4}{|c|}{ 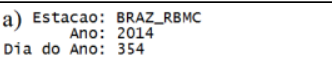 } & \multicolumn{2}{|c|}{$\begin{array}{l}\text { b) Estacaa: BRAZRRBMC } \\
\text { Dia do Ano: } 2014 \\
\text { Ano: } \\
554\end{array}$} & \multicolumn{4}{|c|}{$\begin{array}{l}\text { c) Estacao: } \text { BRAZ_-RBMC } \\
\text { Dia do Ano: } 2014 \text { Ano } 354\end{array}$} & \multicolumn{4}{|c|}{$\begin{array}{l}\text { d) Estacao: } \text { BRAZ_RBMC } \\
\text { Dia do Ano: } 2014 \text { Ano } \\
\text { Dia }\end{array}$} \\
\hline [TU] & [seçäo] & [sat] & {$[\mathrm{fp}]$} & [тU] & {$[\mathrm{Fp}]$} & {$[T \mathrm{U}]$} & [seçāo] & [sat] & [sigma-Phi (L1)] & [тU] & [seçāo] & [sat] & {$[S-P h i(L 1)]$} \\
\hline 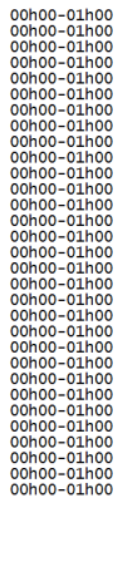 & $\begin{array}{l}{ }_{4}^{4} \\
4 \\
4\end{array}$ & $\begin{array}{l}18 \\
21 \\
22 \\
29 \\
33 \\
40 \\
54 \\
18 \\
21 \\
22 \\
29 \\
33 \\
40 \\
54 \\
55 \\
16 \\
18 \\
21 \\
22 \\
33 \\
40 \\
54 \\
55 \\
14 \\
16 \\
18 \\
22 \\
33 \\
50 \\
54 \\
55\end{array}$ & $\begin{array}{l}0.220 \\
0.277 \\
0.108 \\
0.033 \\
0.090 \\
0.229 \\
0.144 \\
0.122 \\
0.1107 \\
0.174 \\
0.036 \\
0.169 \\
0.101 \\
0.081 \\
0.636 \\
0.352 \\
0.191 \\
0.093 \\
0.201 \\
0.287 \\
0.211 \\
0.184 \\
0.187 \\
0.060 \\
0.19 \\
0.128 \\
0.141 \\
0.137 \\
0.225 \\
0.123\end{array}$ & 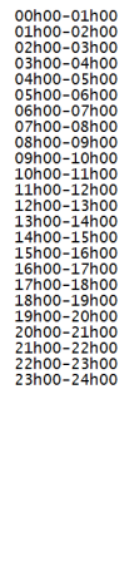 & $\begin{array}{l}161.702 \\
145.862 \\
74.092 \\
32.655 \\
25.253 \\
16.743 \\
12.743 \\
12.623 \\
12.093 \\
12.470 \\
19.489 \\
19.955 \\
22.165 \\
26.165 \\
26.159 \\
22.158 \\
26.886 \\
28.153 \\
29.081 \\
26.065 \\
20.595 \\
20.591 \\
30.093 \\
30.601 \\
30.546 \\
61.193 \\
127.109\end{array}$ & 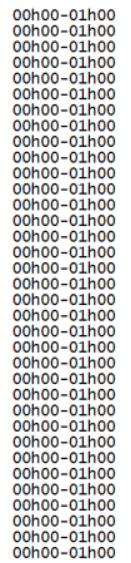 & $\begin{array}{l}02 \\
02 \\
02 \\
02 \\
02 \\
02 \\
02 \\
02 \\
03 \\
03 \\
03 \\
03 \\
03 \\
03 \\
03 \\
03 \\
03 \\
04 \\
04 \\
04 \\
04 \\
04 \\
04 \\
04 \\
04 \\
05 \\
05 \\
05 \\
05 \\
05 \\
05 \\
05 \\
06 \\
06 \\
06 \\
06 \\
06 \\
06 \\
06 \\
06\end{array}$ & $\begin{array}{l}18 \\
21 \\
22 \\
29 \\
33 \\
40 \\
54 \\
18 \\
21 \\
21 \\
22 \\
33 \\
40 \\
54 \\
18 \\
21 \\
22 \\
29 \\
33 \\
40 \\
54 \\
18 \\
21 \\
22 \\
29 \\
33 \\
40 \\
54 \\
18 \\
21 \\
22 \\
29 \\
33 \\
40 \\
54\end{array}$ & $\begin{array}{l}0.036 \\
0.026 \\
0.007 \\
0.001 \\
0.031 \\
0.022 \\
0.024 \\
0.029 \\
0.058 \\
0.058 \\
0.004 \\
0.005 \\
0.028 \\
0.000 \\
0.025 \\
0.032 \\
0.003 \\
0.005 \\
0.005 \\
0.034 \\
0.005 \\
0.025 \\
0.039 \\
0.011 \\
0.005 \\
0.008 \\
0.011 \\
0.028 \\
0.013 \\
0.009 \\
0.012 \\
0.002 \\
0.003 \\
0.031 \\
0.011 \\
0.025\end{array}$ & 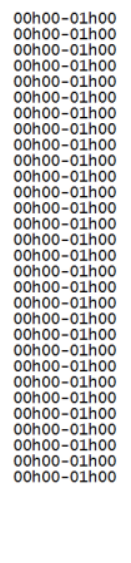 & $\begin{array}{l}02 \\
02 \\
02 \\
02 \\
02 \\
02 \\
02 \\
03 \\
03 \\
03 \\
03 \\
03 \\
03 \\
03 \\
03 \\
03 \\
04 \\
04 \\
04 \\
04 \\
04 \\
04 \\
04 \\
04 \\
05 \\
05 \\
05 \\
05 \\
06 \\
06 \\
06 \\
06 \\
06 \\
06 \\
06\end{array}$ & $\begin{array}{l}18 \\
22 \\
29 \\
33 \\
40 \\
54 \\
18 \\
21 \\
22 \\
29 \\
33 \\
40 \\
54 \\
18 \\
21 \\
22 \\
29 \\
33 \\
40 \\
54 \\
22 \\
29 \\
33 \\
18 \\
18 \\
22 \\
33 \\
40 \\
54\end{array}$ & 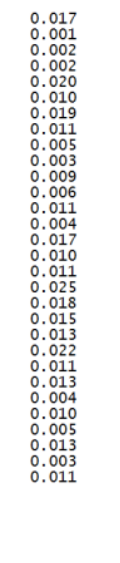 \\
\hline
\end{tabular}

Figura 8: Valores dos índices a) $f_{P}$, b) $F_{P}$, c) $\sigma_{\Phi}$ e d) $S_{\Phi}$, BRAZ/RBMC, 20/12/2014.

Para a validação dos índices de irregularidades foram estimados índices para a estação BOAV (Boa Vista/RR) da RBMC para o dia 28 de fevereiro de 2014 (Figura 9), dia esse caracterizado por fortes níveis de irregularidades da ionosfera devido ao aumento da variação da densidade eletrônica próximo ao equinócio de outono e de uma incursão negativa do índice Dst, que oscilou entre 0 e -100 nT, bem como obtidos os parâmetros ionosféricos h'F (altura virtual mínima do traço F) e hmF2 (altura do pico de densidade eletrônica da camada F2) da ionossonda digital de Boa Vista do INPE (Instituto Nacional de Pesquisas Espaciais). Além disso, foi 
estimada a densidade máxima de elétrons da camada F2 (NmF2) a partir dos valores de foF2 (frequência crítica da onda ordinária da camada F2) (Figura 10).

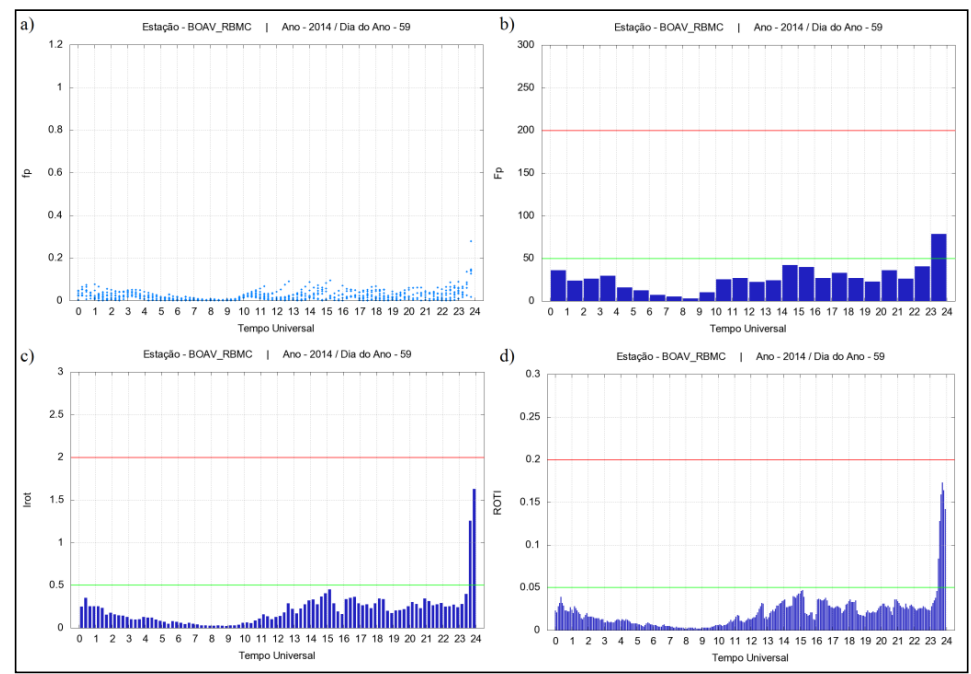

Figura 9: Gráficos dos índices a) fP, b) $F_{P}$, c) IROT e d) ROTI, BOAV/RBMC, 28/02/2014.

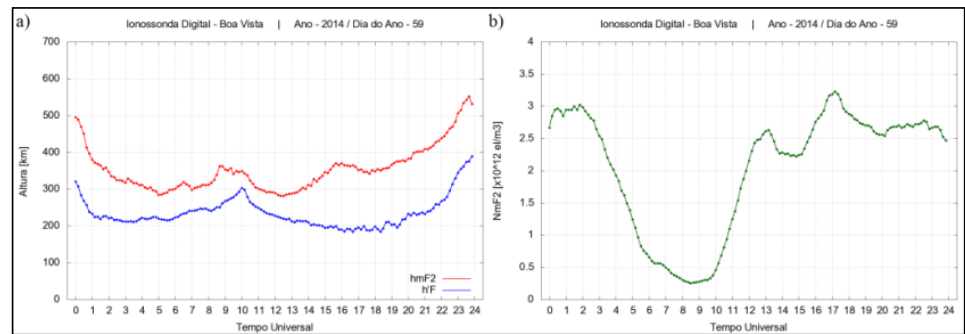

Figura 10: Gráficos dos parâmetros a) h'F, hmF2 e b) NmF2, ionossonda Boa Vista/INPE, $28 / 02 / 2014$.

Comparando os instantes em que os valores dos índices de irregularidades se elevaram na Figura 9 com os respectivos momentos dos parâmetros ionosféricos h'F e hmF2 da Figura 10a, observase uma concordância temporal. Nos períodos $00-01 \mathrm{~h}$ e $23-24 \mathrm{~h}$ TU a base da camada F ionosférica teve elevações acentuadas ( $h^{\prime} \mathrm{F}=321 \mathrm{~km}$ e h'F $=390 \mathrm{~km}$, respectivamente), suficiente para iniciar os processos de geração de irregularidades ionosféricas com escalas de tamanho capazes de gerar flutuações na fase dos sinais GNSS, corroborado pelo aumento das alturas do pico de ionização $(\mathrm{hmF} 2=495 \mathrm{~km}$ e hmF2 $=542 \mathrm{~km})$. Para os demais períodos, os valores da altura do pico de ionização (hmF2) juntamente com a base da camada $\mathrm{F}$ (h'F) permaneceram relativamente baixos, em torno de $\mathrm{hmF} 2=285 \mathrm{~km} \mathrm{e} \mathrm{h'F}=210 \mathrm{~km}$, respectivamente. Estas irregularidades não tinham amplitudes capazes de causar flutuações nos sinais GNSS, o que pode ser verificado nos valores dos índices de irregularidades.

Coeficientes de correlação de Pearson foram calculados com os índices de irregularidades e os parâmetros ionosféricos para o período de alta variabilidade da densidade de elétrons. Entre os índices de irregularidades e o parâmetro h'F a correlação foi em torno de $42 \%$, com o parâmetro hmF2 aproximadamente $62 \%$, e com a densidade eletrônica máxima da camada F2 próximo de $71 \%$, o que mostra uma correlação moderada entre os valores.

Visando comprovar a estimativa dos índices $f_{P}, F_{P}$, IROT e ROTI para um período de baixas irregularidades ionosféricas foram obtidos índices para a estação CEFT (Fortaleza/CE), para o dia 11 de junho de 2014, próximo ao solstício de inverno (menor variabilidade da densidade de 
elétrons). A Figura 11 apresenta os índices de irregularidades e a Figura 12 os valores de h'F, hmF2 e NmF2 da ionossonda de Fortaleza.

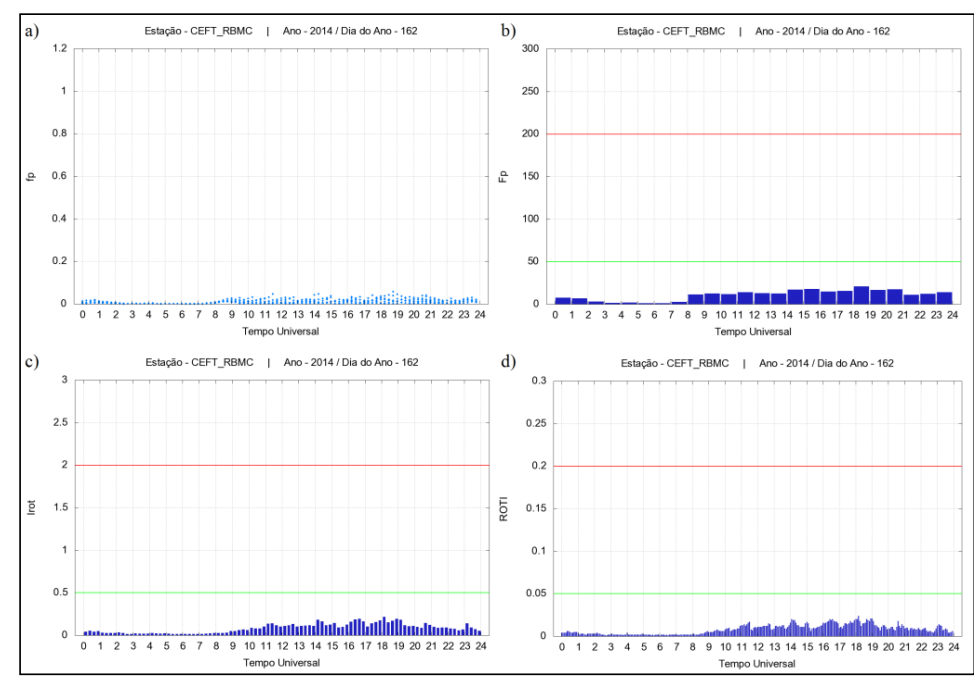

Figura 11: Gráficos dos índices a) $f_{P}$, b) $F_{P}$, c) IROT e d) ROTI, CEFT/RBMC, 11/06/2014.

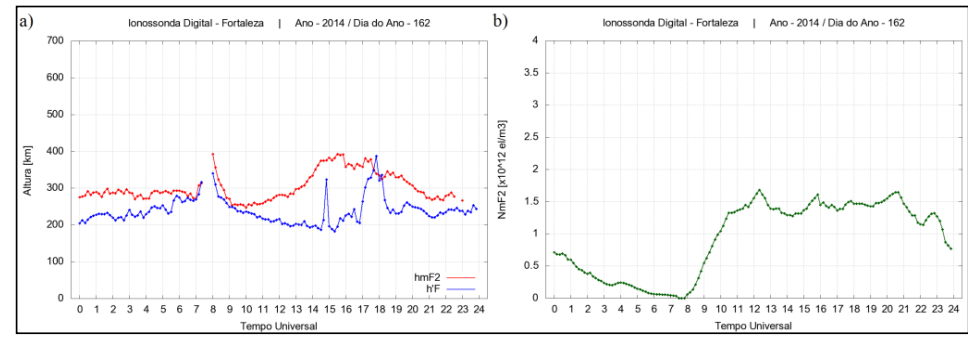

Figura 12: Gráficos dos parâmetros a) h'F, hmF2 e b) NmF2, ionossonda Fortaleza/INPE, $11 / 06 / 2014$.

Neste caso todos os valores dos indicadores foram inferiores aos limiares de baixos níveis de irregularidades da ionosfera (linha verde da Figura 11), corroborado pelos baixos valores de h' $F$ (em torno de $210 \mathrm{~km}), \mathrm{hmF} 2(270 \mathrm{~km})$ e $\mathrm{NmF} 2\left(1,4 \times 10^{12} \mathrm{el} / \mathrm{m}^{3}\right)$, o que indica a correspondência entre a classificação das irregularidades por meio dos índices e por meio dos parâmetros ionosféricos. Os coeficientes de correlação obtidos oscilaram em torno de 54\% para h'F e hmF2 e próximo de $91 \%$ para $\mathrm{NmF} 2$.

Assim avalia-se que a estimativa dos índices de irregularidades da ionosfera pelo programa é coerente com a realidade, uma vez que os valores dos índices foram comparados com dados de fonte externa. No entanto, ressalta-se que foram utilizados os valores de apenas duas ionossondas digitais (Boa Vista e Fortaleza) do INPE, haja vista que apenas ambas estavam operantes nos períodos em análise.

Para a avaliação dos índices de cintilações de fase $\left(\sigma_{\Phi}, S_{\Phi}\right.$ e $\left.\sigma_{\text {CHAIN }}\right)$ foi considerado o mesmo dia de alta variação da densidade de elétrons da ionosfera utilizado para a avaliação das irregularidades (28 de fevereiro de 2014) e o dia 7 de junho de 2014, onde há uma diminuição da variabilidade da densidade eletrônica devido à variação sazonal do ângulo zenital do Sol.

Índices de cintilações de fase foram estimados para seis estações da rede CALIBRA: INCO, PALM, POAL, PRU1, SJCE e UFBA. Para a estimativa o programa utiliza as medidas de fase da onda portadora contidas nos arquivos RINEX de observações das estações (amostragem de $0,067 \mathrm{~Hz}$ ). Para essas mesmas estações e dias foram obtidos os gráficos do índice $\sigma_{\Phi}$ por meio da ferramenta web ISMR Query Tool. O ISMR Query Tool permite a consulta aos valores dos 
índices S4 e $\sigma_{\Phi}$ das estações da rede CALIBRA, sendo que esses valores são disponibilizados pelos receptores PolaRxS-PRO da Septentrio por meio de cálculos e aplicação de método de detrending apropriado aos dados brutos, os quais estão amostrados em $50 \mathrm{~Hz}$. Assim, para cada estação selecionada, têm-se o índice $\sigma_{\Phi}$ proveniente do ISMR Query Tool, o qual no trabalho é considerado o "valor verdadeiro/conhecido", e o índice $\sigma_{\Phi}$ do Ion_Index, considerado o "valor estimado", bem como, os outros dois índices de cintilações de fase $\bar{S}_{\Phi}$ e $\sigma_{C H A I N}$. Dessa forma, os resultados foram comparados com o intuito de avaliar a estimativa dos índices de cintilações de fase.

Nas Figuras 13 e 14 apresentam os índices $\sigma_{\Phi}, S_{\Phi}$ e $\sigma_{C H A I N}$ para a portadora L1 estimados pelo programa e o índice $\sigma_{\Phi}$ dado pelo ISMR Query Tool, das estações PRU1 e UFBA, para o dia 28 de fevereiro de 2014; e as Figuras 15 e 16 apresentam os mesmos índices para o dia 7 de junho de 2014.

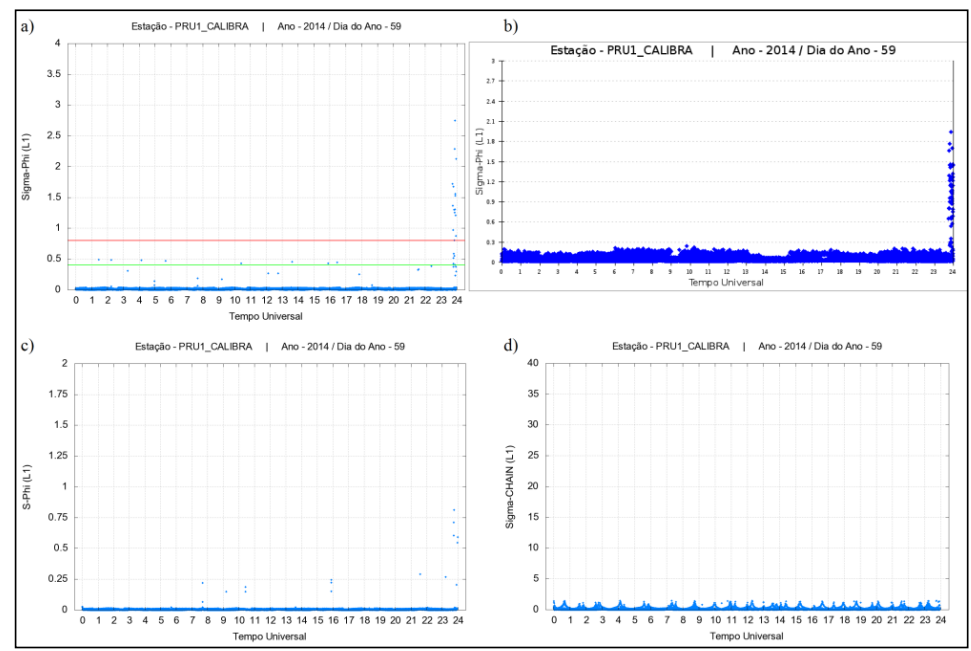

Figura 13: Gráficos dos índices a) $\sigma_{\Phi}, c$ ) $S_{\Phi}, d$ ) $\sigma_{\text {CHAIN }}$ (Ion_Index) e b) $\sigma_{\Phi}$ (ISMR Query Tool), PRU1/CALIBRA, 28/02/2014.

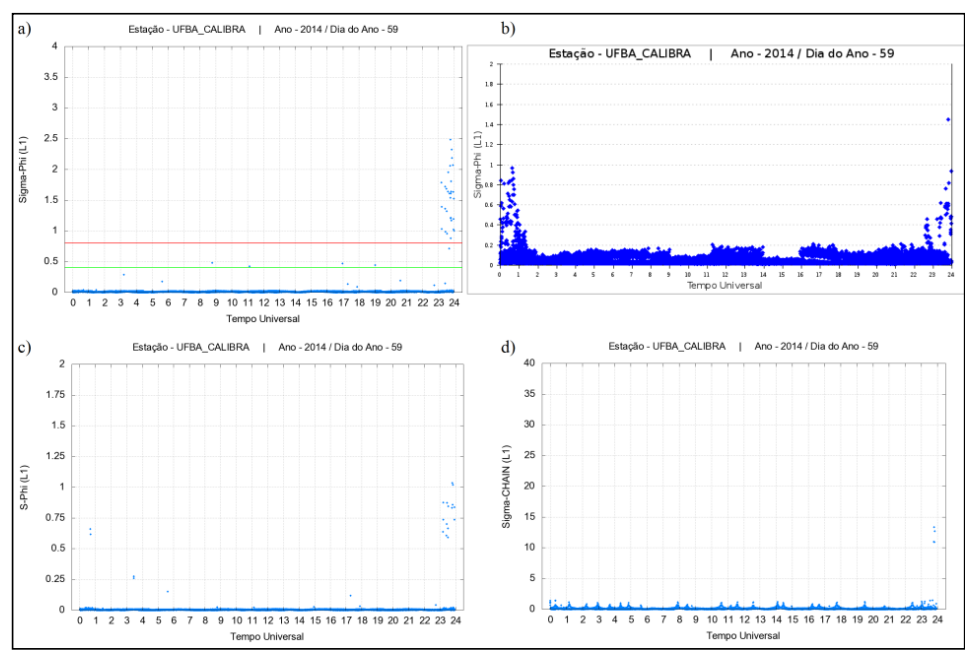

Figura 14: Gráficos dos índices a) $\sigma_{\Phi}$, c) $S_{\Phi}, d$ ) $\sigma_{\text {CHAIN }}\left(\right.$ Ion_Index) e b) $\sigma_{\Phi}($ ISMR Query Tool), UFBA/CALIBRA, 28/02/2014. 


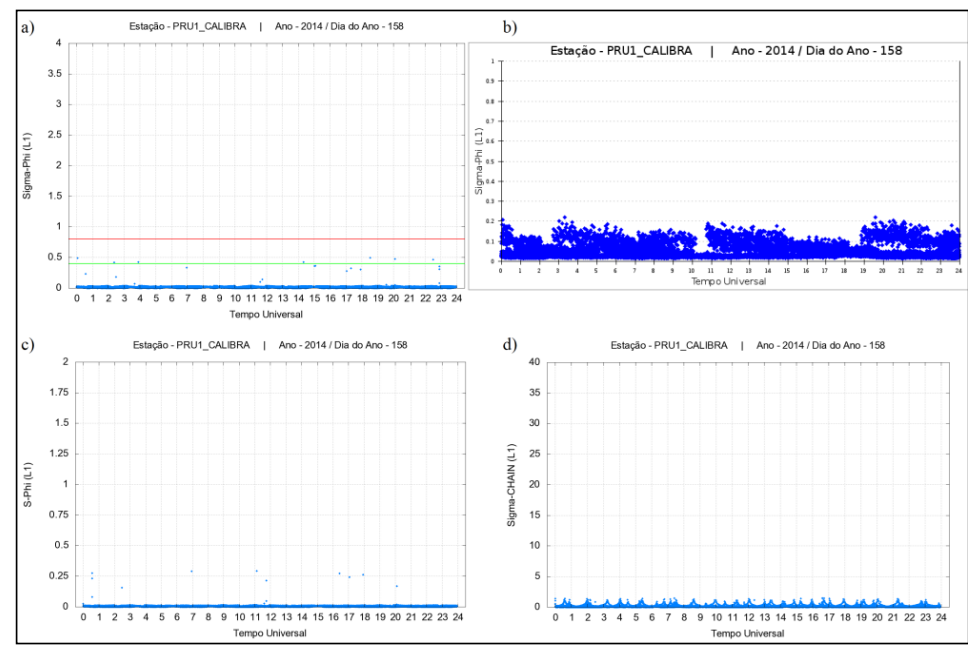

Figura 15: Gráficos dos índices a) $\sigma_{\Phi}$, c) $S_{\Phi}, d$ ) $\sigma_{\text {CHAIN }}\left(\right.$ Ion_Index) e b) $\sigma_{\Phi}$ (ISMR Query Tool), PRU1/CALIBRA, 07/06/2014.

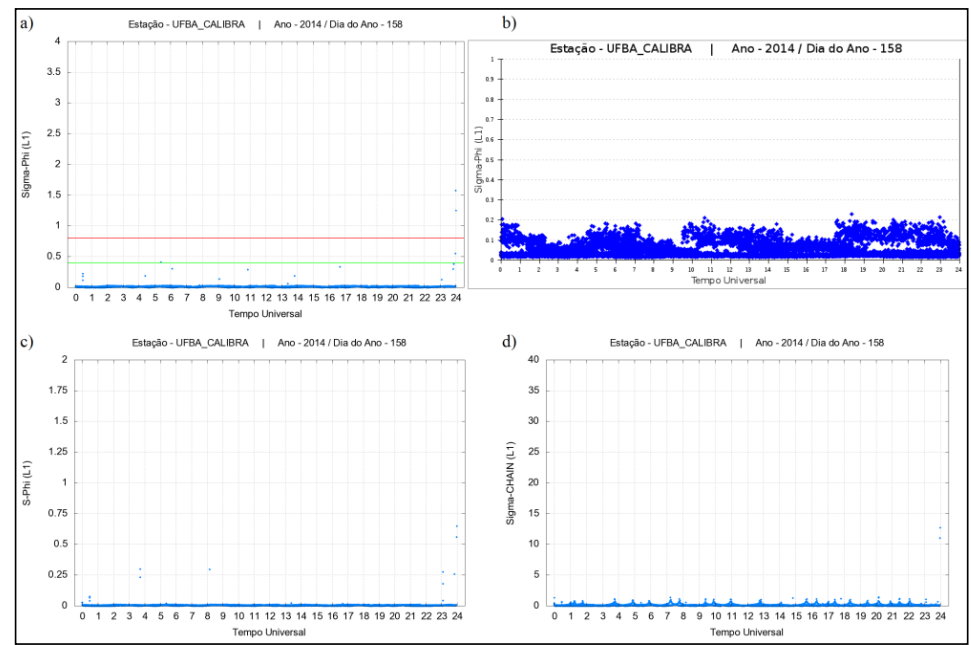

Figura 16: Gráficos dos índices a) $\sigma_{\Phi}$, c) $S_{\Phi}, d$ ) $\sigma_{\text {CHAIN }}\left(\right.$ Ion_Index) e b) $\sigma_{\Phi}$ (ISMR Query Tool), UFBA/CALIBRA, 07/06/2014.

Considerando apenas os valores do índice $\sigma_{\Phi}$ proveniente dos receptores de monitoramento da ionosfera (gráfico b das Figuras 13 a 16) e os valores estimados pelo programa (gráfico a das Figuras 13 a 16) verifica-se que, para ambos os dias em questão, o comportamento dos valores foram semelhantes, isto é, na ocorrência de cintilação ionosférica os valores estimados identificaram o evento no mesmo instante e com a mesma duração que os valores conhecidos, bem como, na ausência de cintilação, onde os valores estimados e conhecidos permaneceram abaixo do limiar de classificação de baixa cintilação. Ressalta-se que os valores de $\sigma_{\Phi}$ estimados não apresentam diferenças significantes daqueles fornecidos pelos receptores. Observa-se que durante a ausência de cintilação ionosférica, os valores do índice $\sigma_{\Phi}$ estimados são inferiores àqueles fornecidos pela ferramenta $w e b$, ao contrário da situação em que ocorre cintilação, cujos valores são superiores aos considerados verdadeiros. Esse comportamento dos valores estimados se justifica pela metodologia aplicada para realizar o detrending das medidas da fase da onda portadora, bem como, devido à taxa de amostragem dos dados. No caso dos valores de $\sigma_{\Phi}$ proveniente do ISMR Query Tool, a amostragem das medidas de fase é $50 \mathrm{~Hz}$, ou seja, há 50 medidas de fase a cada segundo, totalizando 3.000 medidas para determinar o desvio-padrão, além da aplicação do método de filtragem Butterworth para realizar o detrending. Já no Ion_Index a amostragem é $0,067 \mathrm{~Hz}$ - 1 observação a cada 15 segundos - o que proporciona 
apenas 5 medidas para o cálculo do desvio-padrão, e o detrending se resume em controlar o multicaminho dos sinais, através da aplicação de uma máscara de elevação $\left(35^{\circ}\right)$, e detectar as perdas de ciclos. Isso justifica a baixa correlação obtida, para o período de alta variabilidade da densidade eletrônica, entre o índice $\sigma_{\Phi}$ verdadeiro e o $\sigma_{\Phi}$ estimado, em média de $55 \%$ considerando as seis estações. Já para os índices $S_{\Phi}$ e $\sigma_{\text {CHAIN }}$ em relação à $\sigma_{\Phi}$ verdadeiro foi de $38 \%$ e $25 \%$, respectivamente. Entretanto, para o dia de baixa variabilidade da densidade de elétrons, as correlações determinadas foram elevadas, em torno de $76 \%, 65 \%$ e $60 \%$ entre $\sigma_{\Phi}$ verdadeiro com $\sigma_{\Phi}$ estimado, $\mathrm{S}_{\Phi}$ e $\sigma_{\mathrm{CHAIN}}$, respectivamente.

Assim, a possibilidade de identificar a ocorrência de cintilação ionosférica, a duração e uma estimativa da sua intensidade a partir da utilização da fase da onda portadora puderam ser analisadas e avaliadas positivamente, uma vez que há concordância entre os valores, sendo que para todas as situações em que a cintilação ionosférica foi classificada como forte pelo índice conhecido, o índice estimado também apresentou a mesma classificação.

Em relação ao modo tempo real do Ion_Index (Ion_Index_TR) é fornecido a visualização instantânea das irregularidades ionosféricas e cintilação de fase dos sinais GNSS. A Figura 17 apresenta as irregularidades ionosféricas estimadas pelo índice f para o instante $22 \mathrm{~h} 47 \mathrm{~min} 16 \mathrm{~s}$ TU do dia 20/12/2014.

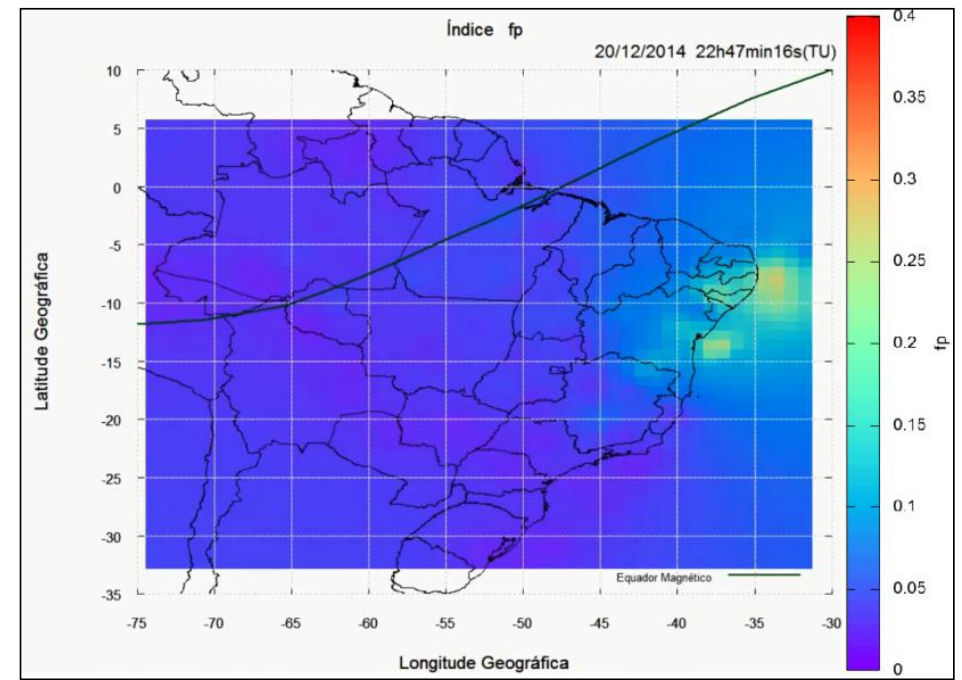

Figura 17: Geovisualização em tempo real do índice $f_{P}$, instante 22h47min16s TU, 20/12/2014.

Pode-se verificar que as irregularidades ionosféricas mostradas a partir do índice fp estimado em tempo real (Figura 17) e pós-processado (Figura 4) apresentam correspondências espacial e temporal. O mesmo ocorreu para os demais índices de irregularidades ( $\mathrm{F}_{\mathrm{P}}, \mathrm{I}_{\mathrm{ROT}}$ e ROTI), bem como para os índices de cintilação ( $\sigma_{\Phi}, \mathrm{S}_{\Phi}$ e $\left.\sigma_{\text {CHAIN }}\right)$.

\section{Conclusões}

O estudo e monitoramento da ionosfera utilizando equipamentos especializados, como ionossondas, imageadores, radares de espalhamento coerente ou receptores geodésicos do tipo PolaRxS-PRO da Septentrio, é forma mais apropriada que existe atualmente, no entanto, em função dos custos, associados aos de manutenção inviabilizam a implantação de uma densa rede ao longo dos 8,5 milhões de $\mathrm{km}^{2}$ de território nacional. Assim, o Ion_Index serve como 
alternativa para o adensamento de informações sobre as irregularidades e cintilação da ionosfera, pois utiliza a infraestrutura de estações já implantadas pela RBMC, GNSS-SP e CALIBRA. Esse adensamento permite uma melhor compreensão da dinâmica dos principais efeitos ionosféricos no Brasil, contribuindo dessa forma para o desenvolvimento ou aprimoramento de modelos de mitigação, principalmente para as técnicas GNSS de alta acurácia, como o PPP (Posicionamento por Ponto Preciso), o RTK (Real Time Kinematic) e o posicionamento relativo estático.

Como recomendações sugere-se a realização de experimentos com o objetivo de avaliar a performance do PPP ou do posicionamento relativo em períodos de irregularidades e cintilações ionosféricas (comprovados por meio dos índices), considerando as mínimas e máximas variabilidades da atividade ionosférica.

\section{AGRADECIMENTOS}

Os autores agradecem à FAPESP (processo 2013/19147-1) e a CAPES pelo apoio financeiro por meio da bolsa de mestrado, ao CNPq (processos 479965/2013-7 e 309924/2013-8) pelo auxílio a pesquisa e bolsa PQ, e ao IBGE, projeto CALIBRA e Laboratório de Geodésia Espacial pelo fornecimento dos dados GNSS das redes ativas.

\section{REFERÊNCIAS BIBLIOGRÁFICAS}

Blewitt, Geoffrey. "An automatic editing algorithm for GPS data." Geophysical Research Letters 17.3 (1990): 199-202.

Camargo, Paulo de Oliveira; Monico, João Francisco Galera, and Ferreira, Luiz Danilo Damasceno. "Application of ionospheric corrections in the equatorial region for L1 GPS users." Earth, Planets and Space 52.11 (2000): 1083-1089.

Chu, F. D., et al. "A study of long-term climatology of ionospheric irregularities by using GPS phase fluctuations at the Brazilian longitudes." Advances in Space Research 41.4 (2008): 645649.

Conker, Robert S., et al. "Modeling the effects of ionospheric scintillation on GPS/Satellite-Based Augmentation System availability.” Radio Science 38.1 (2003): 1-1.

Davies, Kenneth. Ionospheric radio. No. 31. IET, 1990.

Forte, Biagio. "On the relationship between the geometrical control of scintillation indices and the data detrending problems observed at high latitudes." Annals of Geophysics 50.6 (2007): 699-706.

Fortes, Luiz Paulo Souto. Operacionalização da Rede Brasileira de Monitoramento Contínuo do Sistema GPS (RBMC). Diss. Instituto Militar de Engenharia, 1997.

$\mathrm{Fu}$, Wan-xuan, et al. "Some results on the nonparametric statistics of GPS signal scintillation." Intern. Symp. on GPS: Application to Earth Sciences and Interaction with other Space Geodetic Techniques, Tsukuba, Japan. 1999.

Hegarty, Christopher, et al. "Scintillation modeling for GPS-wide area augmentation system receivers." Radio Science 36.5 (2001): 1221-1231. 
Hofmann-Wellenhof, Bernhard, Lichtenegger, Herbert, and Wasle, Elmar. GNSS-global navigation satellite systems: GPS, GLONASS, Galileo, and more. Springer Science \& Business Media, 2008.

Klobuchar, John. "Ionospheric effects on GPS." Global Positioning System: Theory and applications. 1 (1996): 485-515.

Leick, Alfred. GPS satellite surveying. 2. ed. New York: John Wiley \& Sons, 1995. 560 p.

Lin, L. S. "A novel approach to improving the accuracy of real-time ionospheric delay estimation using GPS." Proceedings of The 10th International Technical Meeting of the Satellite Division of The Institute of Navigation-ION GPS. Vol. 97. 1997.

Matsuoka, Marcelo Tomio, and Camargo, Paulo de Oliveira. "Cálculo do TEC usando dados de receptores GPS de dupla frequência para a produção de mapas da ionosfera para a região brasileira." Revista Brasileira de Cartografia 1.56 (2004).

Mendillo, Michael, Lin, Bosheng, and Aarons, Jules. "The application of GPS observations to equatorial aeronomy." Radio Science 35.3 (2000): 885-904.

Monico, João Francisco Galera. Posicionamento pelo GNSS: descrição, fundamentos e aplicações. 2. ed. São Paulo: Editora UNESP, 2008. 476 p.

Mushini, Sajan C., et al. "Improved amplitude-and phase-scintillation indices derived from wavelet detrended high-latitude GPS data." GPS solutions 16.3 (2012): 363-373.

Niu, Fei. Performances of GPS signal observables detrending methods for ionosphere scintillation studies. Diss. Miami University, 2012.

Oladipo, O. A., and Schüler, Torben. "Equatorial ionospheric irregularities using GPS TEC derived index." Journal of Atmospheric and Solar-Terrestrial Physics 92 (2013): 78-82.

Pereira, Vinícius Amadeu Stuani, and Camargo, Paulo de Oliveira. "Estimativa e análise de índices de irregularidades da ionosfera utilizando dados GPS de redes ativas." Bol. Ciênc. Geod., sec. Artigos 19.3 (2013): 374-390

Pereira, Vinícius Amadeu Stuani, and Camargo, Paulo de Oliveira. "Padrões para classificação dos índices de irregularidades da ionosfera: IROT e ROTI." Anais do V Simpósio Brasileiro de Ciências Geodésicas e Tecnologias da Geoinformação, Instituto de Tecnologia de Pernambuco, Universidade Federal de Pernambuco, Recife, 12-14 nov. 2014.

$\mathrm{Pi}$, X., et al. "Monitoring of global ionospheric irregularities using the worldwide GPS network." Geophysical Research Letters 24.18 (1997): 2283-2286.

Rodrigues, Fabiano da S. Estudo das irregularidades ionosféricas equatoriais utilizando GPS. Diss. Instituto Nacional de Pesquisas Espaciais, 2003.

Seeber, Günter. Satellite geodesy: foundations, methods, and applications. Walter de Gruyter, 2003.

Shan, S. J., et al. "GPS phase fluctuations observed along the American sector during low irregularity activity months of 1997-2000." Earth, planets and space 54.2 (2002): 141-152.

Van Dierendonck, A. J., Klobuchar, John, and Hua, Quyen. "Ionospheric scintillation monitoring using commercial single frequency C/A code receivers." proceedings of ION GPS. Vol. 93. 1993.

Walter, T. "Effect of ionospheric scintillations on GNSS - a white paper." SBAS Ionospheric Working Group (2010). 
Wanninger, Lambert. "Ionospheric monitoring using IGS data." Proceedings of the 1993 IGS Workshop. 1993.

Wanninger, Lambert. "Monitoring ionospheric disturbances using the IGS network." SPECIAL TOPICS AND NEW DIRECTIONS (1995): 57.

Webster, Iain R. A regional model for prediction of ionospheric delay for single frequency users of the Global Positioning System. Thesis University of New Brunswick, 1993.

Recebido em junho de 2015.

Aceito em outubro de 2015. 